\title{
Pacific
}

Journal of

Mathematics

\section{THE ENDLICH BAER SPLITTING PROPERTY}

THEODORE GERARD FATICONI

Volume $157 \quad$ No. 2 


\title{
THE ENDLICH BAER SPLITTING PROPERTY
}

\author{
THEODORE G. FAticonI
}

It is well known that projective modules $P$ are characterized by the property that each surjection $M \rightarrow P$ of modules splits. For arbitrary modules $A$ one can ask for conditions under which each surjection $A^{(c)} \rightarrow A^{(d)}$ will split where $c$ and $d$ are cardinals. Modules with this property are said to have the Baer splitting property. If the surjection $A^{(c)} \rightarrow A^{(d)}$ splits whenever $d$ is a finite cardinal then $A$ is said to have the finite Baer splitting property. If the surjection $A^{(c)} \rightarrow A^{(d)}$ splits whenever $c$ and $d$ are finite cardinals then $A$ is said to have the endlich Baer splitting property. Albrecht generalizes a theorem of Arnold and Lady by showing that if $A$ satisfies mild hypotheses, then $A$ has the Baer splitting property iff $I A \neq A$ for each proper right ideal $I \subset \operatorname{End}(A)$.

The goal of this paper is to organize what is known about the (finite, endlich) Baer splitting property by generalizing to pairs $(A, P)$ that have the (endlich) Baer splitting property. (See definitions below.) As an application, we show that the torsion-free abelian group of finite rank has the finite Baer splitting property iff it has the endlich Baer splitting property. We cite examples to show that this result is not true of countable modules.

1. Introduction. Throughout this paper, $R$ denotes a fixed but otherwise arbitrary associative ring, $A$ is a right $R$-module, and $E=$ $\operatorname{End}_{R}(A)$ denotes the ring of $R$-module endomorphisms of $A$. The term module will mean right $R$-module, $\mathscr{M}_{R}$ denotes the category of modules, and $\mathscr{M}_{E}$ denotes the category of right $E$-modules. Let $T_{A}(\cdot)=\cdot \otimes_{E} A$ and let $H_{A}(\cdot)=\operatorname{Hom}_{R}(A, \cdot)$. The module $G$ is (finitely) $A$-generated if there is a (finite) subset $H \subset H_{A}(G)$ such that $G=\sum\{f(A) \mid f \in H\}$.

Fix a pair $(A, P)$ of modules, and consider the statements

(I) If $g: G \rightarrow P$ is a surjection of modules such that $G^{\prime}+\operatorname{ker} g=G$ for some $A$-generated submodule $G^{\prime} \subset G$, then $g$ is a split surjection.

$\left(\mathbf{I}_{0}\right)$ If $g: G \rightarrow P$ is a surjection of modules such that $G^{\prime}+\operatorname{ker} g=$ $G$ for some finitely $A$-generated submodule $G^{\prime} \subset G$, then $g$ is a split surjection.

Reinhold Baer has proved that if $A$ is a subgroup of the abelian group $\mathbf{Q}$ of rational numbers then $(A, A)$ satisfies (I), [15, Proposition 86.5]. This result, known as Baer's Lemma, has assumed an 
important role in the study of the transfer of properties between abelian groups and their endomorphism rings.

For example, $(\mathrm{I})$ is used to characterize the modules with Noetherian hereditary endomorphism rings $[3,7,13],\left(I_{0}\right)$ is used to characterize the modules with semi-hereditary endomorphism rings, $[13,18]$, and Hausen [18] uses $\left(\mathrm{I}_{0}\right)$ to study modules $A$ with the finite summand intersection property. See the references and 8.1 for more examples.

The module $P$ is finitely A-projective if $P$ is a direct summand of $A^{(n)}$ for some integer $n$. Following [4] we say that $A$ has the finite Baer splitting property if $(A, P)$ satisfies (I) for each finitely $A$ projective module $P$. We further specialize this concept as follows. The pair $(A, P)$ of modules has the (endlich) Baer splitting property if it satisfies (I) (if it satisfies $\left(\mathrm{I}_{0}\right)$ ). Fix the module $A$. If $(A, P)$ has the endlich Baer splitting property for each finitely $A$-projective module $P$ then we say that $A$ has the endlich Baer splitting property.

Now consider the properties

(II) $I A \neq A$ for each proper right ideal $I \subset E$,

$\left(\mathrm{II}_{0}\right) \quad I A \neq A$ for each proper finitely generated right ideal $I \subset E$.

Examples of modules that satisfy (II) and $\left(\mathrm{II}_{0}\right)$ include the torsionfree abelian groups $A$ of finite rank such that $E$ is either (semi-) hereditary, $[3,7,13,18]$, (sub-)commutative [7, 13], or local [13]. Arnold and Lady [7, Theorem 2.1] show that the torsion-free abelian group $A$ of finite rank has the finite Baer splitting property iff it satisfies (II). Albrecht [2, Corollary 2.2] extends this result by showing that the self-small module $A$ has the finite Baer splitting property iff $A$ satisfies

(III) $T_{A}(M) \neq 0$ for each nonzero finitely generated $M \in \mathscr{M}_{E}$.

( $A$ is a self-small module if for each cardinal $c$ the canonical imbedding $\operatorname{Hom}_{R}(A, A)^{(c)} \rightarrow \operatorname{Hom}_{R}\left(A, A^{(c)}\right)$ is an isomorphism.) In [13, Propositions 2.3, 2.4] we show that $(A, P)$ has the endlich Baer splitting property iff $A$ satisfies ( $\left.\mathrm{II}_{0}\right)$ iff $K A \neq P$ for each finitely $A$-projective module $P$ and proper finitely generated $E$-submodule $K \subset H_{A}(P)$. It is then natural to consider the property

(III $) T_{A}(M) \neq 0$ for each nonzero finitely presented $M \in \mathscr{M}_{E}$.

Each of the aforementioned results is proved by passing to $E$ via the functors $T_{A}$ and $H_{A}$. The use of the working hypotheses in [2] and [7] leads us to believe that [2, Theorem 2.1] is true for a larger class of modules. Furthermore, the properties (II), $\left(\mathrm{II}_{0}\right),(\mathrm{III})$, and $\left(\mathrm{III}_{0}\right)$ are torsion theoretic in nature, so the logical relationships among them should be proved without invoking the Baer splitting property. 
The goal of this paper is to study the relationship between the above six properties. Our approach will differ from those in [2], [7], and [13] in that we discuss the splitting properties without passing to the endomorphism ring, and we discuss (II) through ( $\mathrm{III}_{0}$ ) without explicitly mentioning the splitting properties. This approach allows us to extend the results from the literature by deleting unnecessary hypotheses.

A detailed description of the sections follows.

In $\S 2$ we prove that the endlich Baer splitting property is passed onto finite direct sums. Thus, $(A, A)$ has the (endlich) Baer splitting property iff $A$ has the finite (endlich) Baer splitting property 2.4, 2.6. The proof avoids the endomorphism ring.

In $\S 3$ we prove (II) $\Rightarrow$ (III) without referring to the Baer splitting property. This extends [2, Corollary 2.2], [7, Theorem 2.1] by removing the self-small hypothesis on $A$. We also prove $\left(\mathrm{II}_{0}\right) \Rightarrow\left(\mathrm{III}_{0}\right)$ which extends [13, Proposition 2.3] and [18, Theorem 2.2].

In $\S 4$ we prove that if $P$ is an $A$-projective module then $(A, P)$ has the Baer splitting property if $K A \neq P$ for each proper $E$-submodule $K \subset H_{A}(P), 4.2$. Thus, (II) is generally a sufficient condition to imply the finite Baer splitting property for $A$.

The pairs $(A, P)$ of modules possessing the endlich Baer splitting property are characterized in $\S 5$. We prove that if $P$ is a finitely $A$ projective module then $(A, P)$ has the endlich Baer splitting property iff $T_{A}(M) \neq 0$ for each nonzero finitely $H_{A}(P)$-presented $M \in \mathscr{M}_{E}$.

In $\S 6$ we reintroduce the self-small hypothesis on $A$, and then extend [2, Corollary 2.2] and [7, Theorem 2.1] to include pairs of modules $(A, P)$. We also give a new proof of [2, Corollary 2.2] in which the use of the self-small hypothesis is minimized, 6.3.

Finally, in $\S 7$ we give an example of a class of modules for which the endlich and finite Baer splitting properties are equivalent. If $A$ is a torsion-free abelian group of finite rank then $A$ has the endlich Baer splitting property iff $A$ has the finite Baer splitting property, 7.2.

We greatfully acknowledge the assistance of Professor C. Vinsonhaler whose insights were used to produce the given proof of 2.3.

We use the notation and terminology given in [5] and [15]. Given $M \in \mathscr{M}_{E}$ and $G \in \mathscr{M}_{R}$ there are natural homomorphisms $\Phi_{M}: M \rightarrow$ $H_{A} T_{A}(M)$ and $\Theta_{G}: T_{A} H_{A}(G) \rightarrow G$ given by $\left[\Phi_{M}(m)\right](a)=m \otimes a$ and $\Theta_{G}(f \otimes a)=f(a)$ for each $m \in M, f \in H_{A}(G)$, and $a \in A$. Furthermore, given a projective right $E$-module $Q$ and a subset $X \subset$ $Q$ then we will identify $X=\Phi_{Q}(X) \subset H_{A} T_{A}(Q)$. We let $M^{*}$ denote the $E$-dual $\operatorname{Hom}_{E}(M, E)$ of the right $E$-module $M$. We also make 
use of

THEOREM 1.1 [7, Theorem 1.1]. Let $A$ be a module.

(a) $H_{A}$ and $T_{A}$ restrict to inverse equivalences between the category of finitely A-projective modules, and the category of finitely generated projective right $E$-modules.

(b) $\Theta_{P}$ and $\Phi_{Q}$ are isomorphisms for each finitely A-projective module $P$ and finitely generated projective right $E$-module $Q$.

2. Direct sums and direct summands. We show that the Baer splitting property is inherited by direct summands and passed onto direct sums.

The first lemma appears in [2].

LEMMA 2.1. Let $A$ and $P$ be modules. The following are equivalent.

(a) $(A, P)$ has the (endlich) Baer splitting property.

(b) Given a surjection $g: G \rightarrow P$ in $\mathscr{M}_{R}$ such that $G$ is a (finitely) A-projective module, then $g$ is a split surjection.

(c) Given a surjection $g: G \rightarrow P$ in $\mathscr{M}_{R}$ such that $G$ is a (finitely) $A$-projective module, then $\operatorname{ker} g$ is a direct summand of $G$.

Lemma 2.2. Let $A$ and $P$ be modules and assume $(A, P)$ has the (endlich) Baer splitting property.

(a) If $P$ is a (finitely) A-generated module then $P$ is a (finitely) A-projective module.

(b) If $P^{\prime}$ is a direct summand of $P$ then $\left(A, P^{\prime}\right)$ has the (endlich) Baer splitting property.

Proof. (a) Is an easy exercise.

(b) Let $g: G \rightarrow P^{\prime}$ be a surjection in $\mathscr{M}_{R}$ such that $G^{\prime}+\operatorname{ker} g=G$ for some (finitely) $A$-generated submodule $G^{\prime} \subset G$. Write $P=P^{\prime} \oplus X$ for some submodule $X \subset P$ and let $\jmath_{P^{\prime}}: P^{\prime} \rightarrow P, p_{P^{\prime}}: P \rightarrow P^{\prime}$ be the canonical injection, projection. Then $g \oplus 1_{X}: G \oplus X \rightarrow P$ is a surjection in $\mathscr{M}_{R}$ such that $\left(G^{\prime} \oplus X\right)+\operatorname{ker}\left(g \oplus 1_{X}\right)=G \oplus X$. Observe that $X$ is a (finitely) $A$-generated module. Because $(A, P)$ has the (endlich) Baer splitting property there is a map $j: P \rightarrow G \oplus X$ such that $\left(g \oplus 1_{X}\right) j=1_{P}$. The usual elementary argument shows that $\jmath=\jmath^{\prime} \oplus 1_{X}$ for some map $\jmath^{\prime}: P^{\prime} \rightarrow G$. Then

$$
g \jmath^{\prime}=p_{P^{\prime}}\left(g \oplus 1_{X}\right)\left(\jmath^{\prime} \oplus 1_{X}\right)_{\jmath_{P^{\prime}}}=p_{P^{\prime}}\left(g \oplus 1_{X}\right) \jmath \jmath_{P^{\prime}}=p_{P^{\prime}} 1_{P} \jmath_{P^{\prime}}=1_{P^{\prime}}
$$

as required by $(b)$. 
Proposition 2.3. Let $A$ be a module and let $\left\{P_{1}, \ldots, P_{n}\right\}$ be a finite set of modules such that $\left(A, P_{i}\right)$ has the endlich Baer splitting property for each $i=1, \ldots, n$. Then $\left(A, \bigoplus_{n=1}^{n} P_{i}\right)$ has the endlich Baer splitting property.

Proof. Let $g: G \rightarrow \bigoplus_{i=1}^{n} P_{i}$ be a surjection such that $G$ is a finitely $A$-projective module. By 2.1 it suffices to show that ker $g$ is a direct summand of $G$.

We proceed by induction on $n$. By hypothesis $\left(A, P_{1}\right)$ has the endlich Baer splitting property. Assume we have shown that $\left(A, \bigoplus_{i=1}^{n-1} P_{i}\right)$ has the Baer splitting property. Let $e_{n}: \bigoplus_{i=1}^{n} P_{i} \rightarrow \bigoplus_{i=1}^{n-1} P_{i}$ be the canonical projection, and let $G_{n}=\operatorname{ker} e_{n} g$. Because $e_{n} g: G \rightarrow$ $\bigoplus_{i=1}^{n-1} P_{i}$ is a surjection, the induction hypothesis yields a direct sum decomposition

$$
G=G_{n}^{\prime} \oplus G_{n}
$$

for some submodule $G_{n}^{\prime} \subset G$.

Notice that $\operatorname{ker} e_{n} g=g^{-1}\left(P_{n}\right)$, so $P_{n}=g\left(G_{n}\right)=\left(1-e_{n}\right) g\left(G_{n}\right)$. By hypothesis $\left(A, P_{n}\right)$ has the Baer splitting property and by (1) $G_{n}$ is a finitely $A$-projective module, so we can write

$$
G_{n}=G_{n}^{\prime \prime} \oplus\left[G_{n} \cap \operatorname{ker}\left(1-e_{n}\right) g\right]
$$

for some submodule $G_{n}^{\prime \prime} \subset G_{n}$. Next, observe that

$$
G_{n} \cap \operatorname{ker}\left(1-e_{n}\right) g=\operatorname{ker} g .
$$

Finally, a combination of (1), (2), and (3) shows that $G=G_{n}^{\prime} \oplus G_{n}^{\prime \prime} \oplus$ ker $g$, which completes the proof.

Let $P$ be an $A$-projective module. If in 2.3 we choose $\left\{P_{1}, \ldots, P_{n}\right\}$ to be distinct copies of $P$ then using 2.2 we have proved

Corollary 2.4. Let $A$ be a module.

(a) The pair $(A, P)$ of modules has the endlich Baer splitting property iff $\left(A, P^{(n)}\right)$ has the endlich Baer splitting property for each integer $n>0$.

(b) $(A, A)$ has the endlich Baer splitting property iff $A$ has the endlich Baer splitting property.

The same argument shows that the Baer splitting property is passed onto direct sums. 
Proposition 2.5. Let $A$ be a module and let $\left\{P_{1}, \ldots, P_{n}\right\}$ be a finite set of modules such that $\left(A, P_{i}\right)$ has the Baer splitting property for each $i=1, \ldots, n$. Then $\left(A, \bigoplus_{i=1}^{n} P_{i}\right)$ has the Baer splitting property.

Corollary 2.6. Let $A$ be a module.

(a) $(A, P)$ has the Baer splitting property iff $\left(A, P^{(n)}\right)$ has the Baer splitting property for each integer $n>0$.

(b) $(A, A)$ has the Baer splitting property iff $A$ has the finite Baer splitting property.

REMARK 2.7. In the proof of [7, Theorem 2.1] the authors leave it to the reader to prove that the torsion-free abelian group $A$ of finite rank has the finite Baer splitting property if $(A, A)$ has the Baer splitting property. In proving [2, Corollary 2.2] the author states that when $A$ is a self-small module, then this implication follows from an induction on the $A$-rank of $P$. (The $A$-rank of $P$ is the least cardinal number $c$ such that $P$ is a direct summand of $A^{(c)}$.) In [18, Theorem 2.2] it is stated that the proof given in [2] works equally well to prove that the module $A$ has the endlich Baer splitting property if $(A, A)$ has the endlich Baer splitting property. Thus 2.4 and 2.6 extend these results and fill a small gap.

3. $\mathscr{T}_{A}$-compressed $E$-modules. We use a divisibility property for finitely generated projective left $E$-modules to characterize the finitely generated $M \in \mathscr{T}_{A}$.

Let $\mathscr{T}_{A}$ denote the class of $M \in \mathscr{M}_{E}$ such that $T_{A}(M)=0$, let $\mathscr{D}(A)$ denote the set of right ideals $I \subset E$ such that $I A=A$. Let $K \subset M$ be an $E$-submodule. If $M / K \in \mathscr{T}_{A}$ then $K$ is called $\mathscr{T}_{A^{-}}$ dense in $M$. Observe that $I \in \mathscr{D}(A)$ iff $I$ is $\mathscr{T}_{A}$-dense in $E$. If $M$ does not contain a proper (finitely generated) $\mathscr{T}_{A}$-dense $E$-submodule then $M$ is (finitely) $\mathscr{T}_{A}$-compressed.

The following lemma contains some elementary facts that we will use throughout this section. The proof is left to the reader.

LemMA 3.1. Let $A$ be a module, and let $M \in \mathscr{M}_{E}$.

(a) $\mathscr{T}_{A}$ is closed under direct sums, homomorphic images, and extensions (i.e. $\mathscr{T}_{A}$ is a torsion class in $\mathscr{M}_{E}$ ).

(b) Let $K \subset K^{\prime} \subset M$ be $E$-submodules, and let $K$ be $\mathscr{T}_{A}$-dense in $M$. Then $K^{\prime}$ is $\mathscr{T}_{A}$-dense in $M$.

(c) If $M$ is (finitely) $\mathscr{T}_{A}$-compressed and if $N$ is a direct summand of $M$ then $N$ is (finitely) $\mathscr{T}_{A}$-compressed. 
The first result shows that (finite) $\mathscr{T}_{A}$-compression is passed onto direct sums.

LEMMA 3.2. Let $Q$ be a finitely generated projective right $E$-module.

(a) $Q$ is finitely $\mathscr{T}_{A}$-compressed iff $Q^{(n)}$ is finitely $\mathscr{T}_{A}$-compressed for each integer $n>0$.

(b) $Q$ is $\mathscr{T}_{A}$-compressed iff $Q^{(n)}$ is $\mathscr{T}_{A}$-compressed for each integer $n>0$.

Proof. (a) Assume $Q$ is finitely $\mathscr{T}_{A}$-compressed. This is the basis for an induction on $n$. Assume that we have shown that $Q^{(n-1)}$ is finitely $\mathscr{T}_{A}$-compressed.

Let $K \subset Q^{(n)}$ be a finitely generated $\mathscr{T}_{A}$-dense $E$-submodule, fix a canonical direct sum decomposition $Q \oplus Q^{(n-1)}=Q^{(n)}$, let $K^{\prime}$ be the projection of $K$ into $Q$, and let $K^{\prime \prime}=K \cap Q^{(n-1)}$. There is an exact sequence

$$
0 \rightarrow \frac{Q^{(n-1)}}{K^{\prime \prime}} \rightarrow \frac{Q^{(n)}}{K} \rightarrow \frac{Q}{K^{\prime}} \rightarrow 0
$$

of right $E$-modules. By 3.1 (a) $Q / K^{\prime} \in \mathscr{T}_{A}$, so $K^{\prime}$ is a finitely generated $\mathscr{T}_{A}$-dense $E$-submodule of $Q$, and hence $K^{\prime}=Q$ by hypothesis. Then (4) implies that

$$
\frac{Q^{(n-1)}}{K^{\prime \prime}} \cong \frac{Q^{(n)}}{K},
$$

so that $K^{\prime \prime}$ is a $\mathscr{T}_{A}$-dense submodule of $Q^{(n-1)}$. Inasmuch as $K$ and $Q^{(n-1)}$ are finitely generated, Schanuel's Lemma shows that $K^{\prime \prime}$ is a finitely generated $E$-submodule of $Q^{(n-1)}$. Then $K^{\prime \prime}=Q^{(n-1)}$ by induction, and therefore $K=Q^{(n)}$. The converse is clear so the proof is complete.

(b) is proved in an analogous manner.

The following result shows that (finite) $\mathscr{T}_{A}$-compression is a generalization of (III) and $\left(\mathrm{III}_{0}\right)$. The right $E$-module $N$ is called finitely $M$-generated if there is a surjection $M^{(n)} \rightarrow N$ for some integer $n$. The right $E$-module $N$ is called finitely $M$-presented if there is an integer $n>0$ and a finitely generated $E$-submodule $K \subset M^{(n)}$ such that $N \cong M^{(n)} / K$.

COROLlaRY 3.3. Let $Q$ be a finitely generated projective right $E$ module.

(a) $Q$ is finitely $\mathscr{T}_{A}$-compressed iff $T_{A}(M) \neq 0$ for each nonzero finitely $Q$-presented $M \in \mathscr{M}_{E}$. 
(b) $Q$ is $\mathscr{T}_{A}$-compressed iff $T_{A}(M) \neq 0$ for each nonzero finitely $Q$-generated $M \in \mathscr{M}_{E}$.

Proof. (a) By 3.2(a) and the definitions, $Q$ is finitely $\mathscr{T}_{A}$-compressed iff $Q^{(n)}$ is finitely $\mathscr{T}_{A}$-compressed for each integer $n>0$ iff $T_{A}(M) \neq$ 0 for each nonzero finitely $Q$-presented $M \in \mathscr{M}_{E}$.

(b) follows in a manner similar to (a) but appeal to 3.2(b) instead of 3.2(a).

The trace ideal of $Q$ in $E$ is $\tau_{Q}=\sum f(Q)$ where the sum is indexed by $f \in \operatorname{Hom}_{E}(Q, E)$. Our characterization of the projective finitely $\mathscr{T}_{A}$-compressed right $E$-modules $Q$ is in terms of $\tau_{Q}$.

Proposition 3.4. Let $A$ be a module, and let $Q$ be a finitely generated projective right $E$-module. The following are equivalent.

(a) $Q$ is not finitely $\mathscr{T}_{A}$-compressed.

(b) There exists a finitely generated $I \in \mathscr{D}(A)$ such that $(E / I) \tau_{Q}=$ $E / I$.

Proof. (a) $\Rightarrow$ (b) Assume there is a finitely generated $\mathscr{T}_{A}$-dense $E$ submodule $K \subset Q$. Let $\left\{x_{1}, \ldots, x_{n}\right\} \subset Q$ map onto a minimal set of generators for $Q / K$, and let $K^{\prime}=K+\sum_{i=2}^{n} x_{i} E$. Then $K^{\prime}$ is a finitely generated $\mathscr{T}_{A}$-dense $E$-submodule of $Q, 3.1(\mathrm{~b})$, and because $\left\{\bar{x}_{1}, \ldots, \bar{x}_{n}\right\}$ is a minimal set of generators of $Q / K^{\prime \prime}, Q / K^{\prime} \in \mathscr{T}_{A}$ is a nonzero cyclic right $E$-module. There is a right ideal $I \in \mathscr{D}(A)$ such that $Q / K^{\prime} \cong E / I$, and Schanuel's Lemma shows that $I$ is finitely generated. Inasmuch as $Q \tau_{Q}=Q$ we have $(E / I) \tau_{Q}=E / I$, which proves (b).

(b) $\Rightarrow$ (a) Assume there is a proper finitely generated $I \in \mathscr{D}(A)$ such that $(E / I) \tau_{Q}=E / I$. Then $E / I=\left(\tau_{Q}+I\right) / I$ so that there is a cardinal $c$ and a surjection $f: Q^{(c)} \rightarrow E / I$. Because $E / I$ is a cyclic right $E$-module we may assume that $c$ is finite. Then $\operatorname{ker} f$ is finitely generated by Schanuel's Lemma. Furthermore, $Q^{(c)} / \operatorname{ker} f \cong$ $E / I \in \mathscr{T}_{A}$, so that $\operatorname{ker} f$ is a proper finitely generated $\mathscr{T}_{A}$-dense $E$ submodule of $Q^{(c)}$, and hence $Q^{(c)}$ is not finitely $\mathscr{T}_{A}$-compressed. Then by $3.1(\mathrm{c}) Q$ is not finitely $\mathscr{T}_{A}$-compressed.

In contrast to 3.4 , the $\mathscr{T}_{A}$-compressed property in $Q$ is characterized in terms of the dual $Q^{*}=\operatorname{Hom}_{E}(Q, E)$ of $Q$. Let $\mathbf{m}_{A}$ denote the set of maximal right ideals $I \in \mathscr{D}(A)$. The left $E$-module $L$ is $\mathbf{m}_{A}$-divisible if $I L=L$ for each $I \in \mathbf{m}_{A}$. 
Proposition 3.5. Let $A$ be a module, let $Q$ be a finitely generated projective right $E$-module. The following are equivalent.

(a) $Q$ is $\mathscr{T}_{A}$-compressed.

(b) $\tau_{Q} \subset I$ for each $I \in \mathbf{m}_{A}$.

(c) $Q^{*}$ is $\mathbf{m}_{A}$-divisible.

Proof. (a) $\Rightarrow$ (b) We prove the contrapositive. Assume there is an $I \in \mathbf{m}_{A}$ such that $\tau_{Q} \not \subset I$, and let $p: E \rightarrow E / I$ be the natural projection map. There is a map $f: Q \rightarrow E$ such that $f(Q) \not \subset I$, and because $I$ is a maximal right ideal of $E, f(Q)+I=E$. Hence $p f: Q \rightarrow E / I$ is a surjection. Furthermore, $E / I \in \mathscr{T}_{A}$, so $\operatorname{ker} p f$ is a proper $\mathscr{T}_{A}$-dense $E$-submodule of $Q$, and hence $Q$ is not $\mathscr{T}_{A^{-}}$ compressed.

(b) $\Leftrightarrow$ (c) It is well known that $I Q^{*}=Q^{*}$ implies $\tau_{Q^{*}} \subset I$, and that $\tau_{Q}=\tau_{Q^{*}}$. (See e.g. [1].) Thus (b) is equivalent to (c).

(b) $\Rightarrow$ (a) Again, we prove the contrapositive. Assume there is an integer $n>0$ and a proper $\mathscr{T}_{A}$-dense $E$-submodule $K \subset Q$. Because $Q$ is finitely generated there is a maximal right $E$-submodule $K \subset K^{\prime} \subset Q$, and by $3.1\left(\right.$ b) $K^{\prime}$ is $\mathscr{T}_{A}$-dense in $Q$. Since $Q / K^{\prime} \neq 0$ is a simple module there is a maximal right ideal $I \subset E$ such that $Q / K^{\prime} \cong E / I$. Since $I$ is then $\mathscr{T}_{A}$-dense in $E, I \in \mathbf{m}_{A}$. Finally, $Q \tau_{Q}=Q$ so $(E / I) \tau_{Q}=E / I \neq 0$. Hence $\tau_{Q} \not \subset I$, which completes the proof.

We are interested in when the projective modules of the form $H_{A}(P)$ are finitely $\mathscr{T}_{A}$-compressed right $E$-modules. The next result shows that the (finitely) $\mathscr{T}_{A}$-compressed property is a generalization of (II) and $\left(\mathrm{II}_{0}\right)$.

Proposition 3.6. Let $A$ be a module and let $P$ be a finitely $A$ projective module. The following are equivalent.

(a) $H_{A}(P)$ is finitely $\mathscr{T}_{A}$-compressed.

(b) $T_{A}(M) \neq 0$ for each nonzero finitely $H_{A}(P)$-presented $M \in \mathscr{M}_{E}$.

(c) $K A \neq P$ for each proper finitely generated $E$-submodule $K \subset$ $H_{A}(P)$.

Proof. (a) $\Leftrightarrow$ (b) follows from 3.3 because $H_{A}(P)$ is a finitely generated projective right $E$-module, 1.1.

(a) $\Leftrightarrow$ (c) Let $K \subset H_{A}(P)$ be an $E$-submodule, let $\imath: K \rightarrow H_{A}(P)$ be the inclusion map, and observe that $\Theta_{P} T_{A}(l): T_{A}(K) \rightarrow P$ has image $K A$. Because $\Theta_{P}$ is an isomorphism, 1.1(b), it follows that $K$ 
is $\mathscr{T}_{A}$-dense in $H_{A}(P)$ iff $0=T_{A}\left(H_{A}(P) / K\right)=\operatorname{coker} T_{A}(l)$ iff $T_{A}(l)$ is a surjection iff $K A=P$.

The $\mathscr{T}_{A}$-compressed $E$-modules of the form $H_{A}(P)$ are characterized in

Proposition 3.7. Let $A$ be a module and let $P$ be a finitely $A$ projective module. The following are equivalent.

(a) $H_{A}(P)$ is $\mathscr{T}_{A}$-compressed.

(b) $T_{A}(M) \neq 0$ for each nonzero finitely $H_{A}(P)$-generated $M \in \mathscr{M}_{E}$.

(c) $K A \neq P$ for each proper $E$-submodule $K \subset H_{A}(P)$.

(d) $\operatorname{Hom}_{R}(P, A)$ is an $\mathbf{m}_{A^{-}}$divisible left E-module.

Proof. (a) $\Leftrightarrow$ (b) is $3.3(\mathrm{~b})$, and (a) $\Leftrightarrow$ (c) follows as in 3.6.

(a) $\Leftrightarrow\left(\right.$ d) By 3.5 it suffices to show that $\operatorname{Hom}_{R}(P, A)$ is the $E$-dual of $H_{A}(P)$. But this follows from the isomorphism $T_{A} H_{A}(P) \cong P$, 1.1(b), and the adjoint isomorphism

$$
\begin{aligned}
\operatorname{Hom}_{R}(P, A) & \cong \operatorname{Hom}_{R}\left(T_{A} H_{A}(P), A\right) \\
& \cong \operatorname{Hom}_{E}\left(H_{A}(P), H_{A}(A)\right)=H_{A}(P)^{*} .
\end{aligned}
$$

If $\mathscr{T}_{A}$ is a hereditary torsion class, (i.e. if $\mathscr{T}_{A}$ is closed under $E$ submodules), then $\mathscr{T}_{A}=\{0\}$ iff $A$ satisfies (III). Thus the following is not without interest.

Corollary 3.8. Let $A$ be a module.

(a) $A$ satisfies $\left(\mathrm{II}_{0}\right)$ iff $A$ satisfies $\left(\mathrm{III}_{0}\right)$.

(b) $A$ satisfies (II) if $A$ satisfies (III).

Proof. (a) follows immediately from 3.6, (from 3.7), since each finitely presented right $E$-module is finitely $H_{A}(A)$-presented.

(b) is proved in a similar manner, but appeal to 3.7 instead of 3.6.

4. Sufficient conditions. The $\mathscr{T}_{A}$-compressed condition provides a test for the (endlich) Baer splitting property.

Lemma 4.1. Let $A$ be a module and let $P$ be a finitely $A$-projective module. If $H_{A}(P)$ is finitely $\mathscr{T}_{A}$-compressed then $(A, P)$ has the endlich Baer splitting property.

Proof. Let $G$ be a finitely $A$-generated module, let $g: G \rightarrow P$ be an epimorphism in $\mathscr{P}_{A}$, and let $M=\operatorname{coker} H_{A}(g)$. By 2.1 it suffices 
to show that $g$ is a split surjection. An application of $T_{A} H_{A}$ to $g$ yields a commutative diagram

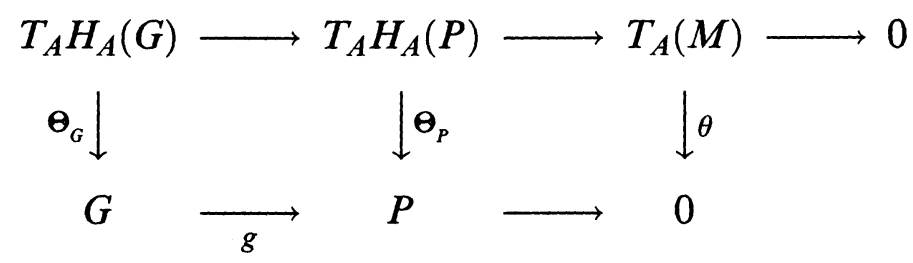

with exact rows in $\mathscr{M}_{R}$. Now $\Theta_{P}$ is an isomorphism, 1.1(b), and because $G$ is a finitely $A$-generated module $\Theta_{G}$ is a surjection. Then a diagram chase shows that $\operatorname{ker} \theta=0$, and hence $T_{A}(M)=0$. Because $H_{A}(P)$ is finitely $\mathscr{T}_{A}$-compressed coker $H_{A}(g)=M=0$, so that $H_{A}(g)$ is a surjection. Since $H_{A}(P)$ is a projective right $E$-module, 1.1(a), there is a map $l: H_{A}(P) \rightarrow H_{A}(C)$ such that $H_{A}(g) l=1_{H_{A}(P)}$. Then

$$
T_{A} H_{A}(\dot{g}) T_{A}(l)=T_{A}\left(1_{H_{A}(P)}\right)=1_{T_{A} H_{A}(P)},
$$

and because the diagram commutes

$$
\left[g \Theta_{G}\right] T_{A}(l) \Theta_{P}^{-1}=\left[\Theta_{P} T_{A} H_{A}(g)\right] T_{A}(l) \Theta_{P}^{-1}=\Theta_{P} 1_{T_{A} H_{A}(P)} \Theta_{P}^{-1}=1_{P} .
$$

Therefore, $(A, P)$ has the endlich Baer splitting property.

A similar argument proves

LemMA 4.2. Let $A$ be a module and let $P$ be an A-projective module such that $H_{A}(P)$ is a projective right $E$-module and such that $\Theta_{P}$ is an isomorphism. If $H_{A}(P)$ is $\mathscr{T}_{A}$-compressed then $(A, P)$ has the Baer splitting property.

Corollary 4.3. Let $A$ be a module.

(a) If $A$ satisfies $\left(\mathrm{II}_{0}\right)$ then $A$ has the endlich Baer splitting property.

(b) If $A$ satisfies (II) then $A$ has the finite Baer splitting property.

Proof. (a) Let $P$ be a finitely $A$-projective module, and let $P \oplus P^{\prime}=$ $A^{(n)}$ for some module $P^{\prime}$ and integer $n>0$. Because $I A \neq A$ for each proper finitely generated right ideal $I \subset E, H_{A}\left(A^{(n)}\right)$ is finitely $\mathscr{T}_{A}$-compressed, 3.6 , so that $H_{A}(P)$ is finitely $\mathscr{T}_{A}$-compressed, $3.1(\mathrm{c})$. Thus $(A, P)$ has the endlich Baer splitting property, 4.1 , and hence $A$ has the endlich Baer splitting property.

(b) Proceed as in part (a).

5. The endlich Baer splitting property. Given a finitely $A$-projective module $P$ let $\tau_{P}$ be the trace ideal of the finitely generated projective right $E$-module $H_{A}(P)$ in $E$. 
Theorem 5.1. Let $A$ be a module, and let $P$ be a finitely $A$ projective module. The following are equivalent for the pair $(A, P)$.

(a) $(A, P)$ has the endlich Baer splitting property.

(b) $H_{A}(P)$ is finitely $\mathscr{T}_{A}$-compressed.

(c) If $E \neq I \in \mathscr{D}(A)$ is finitely generated then $(E / I) \tau_{P} \neq E / I$.

Proof. (b) $\Leftrightarrow$ (c) is 3.4, and (b) $\Rightarrow$ (a) follows from 4.1.

(a) $\Rightarrow$ (b) Let $K \subset H_{A}(P)$ be a finitely generated $\mathscr{T}_{A}$-dense $E$ submodule, and choose a finitely generated projective module $Q$ and a map $k: Q \rightarrow H_{A}(P)$ such that Image $k=K$. Because

$$
\operatorname{coker} T_{A}(k)=T_{A}(\operatorname{coker} k)=T_{A}\left(H_{A}(P) / K\right)=0,
$$

$T_{A}(k): T_{A}(Q) \rightarrow T_{A} H_{A}(P)$ is a surjection in $\mathscr{M}_{R}$. Furthermore, by 1.1(a) $T_{A} H_{A}(P) \cong P$ and $T_{A}(Q)$ is a finitely $A$-projective module, so (a) implies that $T_{A}(k)$ is a split surjection. Finally, an application of $H_{A}$ to $T_{A}(k)$ yields a commutative diagram

$$
\begin{array}{ccc}
Q & \stackrel{k}{\longrightarrow} & H_{A}(P) \\
\Phi_{Q} \downarrow & & \\
H_{A} T_{A}(Q) & \stackrel{\Phi_{H_{A}(P)}}{H_{A} T_{A}(k)} & H_{A} T_{A} H_{A}(P)
\end{array}
$$

in $\mathscr{M}_{E}$ whose bottom row is a (split) surjection. Inasmuch as $\Phi_{Q}$ and $\Phi_{H_{A}(P)}$ are isomorphisms, $1.1(\mathrm{~b}), k$ is a surjection in $\mathscr{M}_{E}$, and hence $K=H_{A}(P)$. This proves (b) and completes the proof.

COROLlary 5.2. The following are equivalent for a module $A$.

(a) $(A, A)$ has the endlich Baer splitting property.

(b) $A$ has the endlich Baer splitting property.

(c) A satisfies $\left(\mathrm{II}_{0}\right)$.

(d) A satisfies $\left(\mathrm{III}_{0}\right)$.

Proof. (a) $\Leftrightarrow$ (b) is $2.4(\mathrm{~b}),(\mathrm{a}) \Leftrightarrow$ (c) is 5.1 , and (c) $\Leftrightarrow$ (d) is 3.8(a).

6. Self-small modules. We extend [2, Corollary 2.2] to include pairs of modules $(A, P)$.

Arnold and Murley [8] prove that if $A$ is a self-small module then $H_{A}$ and $T_{A}$ restrict to inverse equivalences between the category of $A$ projective modules, and the category of projective right $E$-modules. Furthermore, $\Theta_{P}$ and $\Phi_{Q}$ are isomorphisms for each $A$-projective module $P$ and projective right $E$-module $Q$. 
Theorem 6.1. Let $A$ be a self-small module and let $P$ be an $A$ projective module. The following are equivalent for the pair $(A, P)$.

(a) $(A, P)$ has the Baer splitting property.

(b) $H_{A}(P)$ is $\mathscr{T}_{A}$-compressed.

Proof. (a) $\Rightarrow$ (b) Let $K \subset H_{A}(P)$ be a $\mathscr{T}_{A}$-dense $E$-submodule. Proceed as in $5.1(\mathrm{a}) \Rightarrow$ (d) to show that $K=H_{A}(P)$ but appeal to [8] instead of 1.1 to prove that $\Phi_{H_{A}(P)}$ and $\Phi_{Q}$ are isomorphisms. (b) $\Rightarrow(\mathrm{a})$ is 4.2 .

Corollary 6.2. Let $A$ be a self-small module, and let $P$ be a finitely A-projective module. Then $(A, P)$ has the Baer splitting property iff $\operatorname{Hom}_{R}(P, A)$ is $\mathbf{m}_{A}$-divisible.

Proof. Because $P$ is finitely $A$-projective, $\operatorname{Hom}_{R}(P, A)$ is $\mathbf{m}_{A^{-}}$ divisible iff $H_{A}(P)$ is $\mathscr{T}_{A}$-compressed, 3.7, iff $(A, P)$ has the Baer splitting property, 6.1 .

The next result is [2, Corollary 2.2]. The self-small hypothesis is only used to prove (a) $\Rightarrow$ (d).

COROLlARY 6.3. The following are equivalent for a self-small module $A$.

(a) $(A, A)$ has the Baer splitting property.

(b) $A$ has the finite Baer splitting property.

(c) $A$ satisfies (II).

(d) $A$ satisfies (III).

Proof. (d) $\Rightarrow$ (c) is clear, (c) $\Rightarrow($ a) is $4.3(b)$, and (a) $\Rightarrow(b)$ is 2.6(b). (b) $\Rightarrow(\mathrm{d})$ is 6.1 since each $M \in \mathscr{M}_{E}$ is $H_{A}(A)$-generated.

7. Torsion-free Abelian groups. We show that for torsion-free abelian groups of finite rank the endlich Baer splitting property and the finite Baer splitting property are equivalent.

THEOREM 7.1. Let $A$ be a torsion-free abelian group of finite rank, and let $P$ be a finitely $A$-projective module. Then $(A, P)$ has the endlich Baer splitting property iff $(A, P)$ has the Baer splitting property.

Proof. Assume $(A, P)$ has the endlich Baer splitting property and recall that a torsion-free abelian group of finite rank is self-small. Let $I \in \mathbf{m}_{A}$. Then [13, Lemma 3.1a] states that $E / I$ is a finite group, 
so there is an integer $n>0$ such that $n E \subset I$. Since $E / n E$ is bounded and since $E$ has finite rank, $E / n E$ is a finite group. Choose representatives $x_{1}, \ldots, x_{k}$ of the finitely many cosets in $I / n E$, and observe that $\left\{x_{1}, \ldots, x_{k}, n 1_{A}\right\}$ is a finite set of generators for $I$.

Now let $\tau_{P}$ denote the trace ideal of $H_{A}(P)$ in $E$ and let $E \neq$ $I \in \mathbf{m}_{A}$. Because $(A, P)$ has the endlich Baer splitting property and because $E \neq I$ is finitely generated $(E / I) \tau_{P} \neq E / I, 5.1$. Then $\tau_{P} \subset I$ because $I$ is a maximal right ideal of $E$. Thus 3.5 and 6.1 imply that $(A, P)$ has the Baer splitting property. The converse is clear so the proof is complete.

COROLlARY 7.2. The following are equivalent for the torsion-free abelian group $A$ of finite rank.

(a) $A$ has the endlich Baer splitting property.

(b) $A$ has the finite Baer splitting property.

(c) $A$ satisfies $\left(\mathrm{III}_{0}\right)$.

(d) A satisfies (III).

Proof. Use 5.2, 6.3, and 7.1.

\section{Examples.}

REMARK 8.1. (a) Let $c$ be an infinite cardinal and let $A=R^{(c)}$. Then $A$ has the Baer splitting property, and by $5.2 A$ satisfies $\left(\mathrm{III}_{0}\right)$. However, let $\Delta$ be the ideal consisting of the $f \in E$ such that $f(A)$ is a finitely generated module. Then $\Delta$ is a proper ideal of $E$ such that $\Delta A=A$. Thus the converse to 4.3 is not true in general.

(b) $A=\mathbf{Q}$ has the Baer splitting property, but $A=\mathbf{Z}_{p^{\infty}}$ does not have the endlich Baer splitting property.

(c) The main theorems in $[10,11,14]$ show that each cotorsion-free (respectively, countable, reduced torsion-free finite rank) ring $E$ is the endomorphism ring of a cotorsion-free (respectively, countable, reduced torsion-free finite rank) $E$-flat abelian group $A$ that satisfies (II), and hence satisfies $\left(\mathrm{II}_{0}\right)$.

(d) In [14, Example 4.7] there is constructed an $E$-flat torsion-free abelian group $A$ of rank 8 such that $E$ is a (noncommutative) domain, and such that the least right ideal $\Delta$ in $\{I \subset E \mid I A=A\}$ is a proper idempotent ideal of finite index in $E$. Arnold and Lady [7] give an example of a completely decomposable abelian group $A$ of rank 2 such that $I A=A$ for some proper pure ideal $I \subset E$.

(e) Let $\Omega(E)$ be the class of torsion-free abelian groups $A$ of finite rank such that $E \cong \operatorname{End}(A)$. [12, Theorem 7.1] characterizes the 
reduced torsion-free finite rank rings $E$ such that each $A \in \Omega(E)$ satisfies (II).

(f) In [11] there is given an example of a reduced self-small torsionfree abelian group $A$ such that $\mathrm{Z}_{p}[X]=E$, (a countable commutative Noetherian integral domain), $A$ satisfies (II), but $T_{A}(M)=0$ for some nonzero $M \in \mathscr{M}_{E}$. The module $M$ is not finitely generated.

(g) In [11] there is given an example of a reduced self-small torsionfree abelian group $A$ such that $E$ is a countable commutative Noetherian integral domain but $I A=A$ for each of the infinitely many maximal right ideals $I \subset E$.

REMARK 8.2. Statements (II) and (III) appear as working hypotheses in many works concerning properties of endomorphism rings. For example, Arnold [6] calls $A$ finitely faithful if $I A \neq A$ for each maximal right ideal $I$ of finite index in $E$. The module $A$ satisfying statement (III) is called completely faithful by Fuller [16], a weak generator by Azumaya [9] and Sato [19], and fully faithful in [2]. Garcia and Saorin [17] call $A$ intrinsically projective if $K=\operatorname{Hom}_{E}(A, K A)$ for each $E$-submodule $K$ of a finitely generated projective right $E$ module, and Wisbauer [21] calls the module $A$ an ideal module if the assignment $I \mapsto I A$ defines a bijection from the set of right ideals of $E$ onto the set of $A$-generated submodules of $A$. These properties are equivalent to (II) when $A$ is a flat left $E$-module, [13], or a $\Sigma$-quasi-projective module, [17].

\section{REFERENCES}

[1] F. W. Anderson and K. R. Fuller, Rings and Categories of Modules, Graduate texts in Mathematics, vol. 13, Springer-Verlag, New York-Berlin, (1974).

[2] U. Albrecht, Faithful abelian groups of infinite rank, Proc. Amer. Math. Soc., 103 (1) (1988), 21-26.

[3] - Baer's Lemma and Fuch's Problem 84a, Trans. Amer. Math. Soc., 293 (2) (1986), 565-582.

[4] U. Albrecht and H. P. Goeters, A dual to Baer's Lemma, Proc. Amer. Math. Soc., 105 (4) (1989), 817-826.

[5] D. M. Arnold, Finite Rank Torsion-free Abelian Groups and Rings, Lecture Notes in Mathematics, vol. 931, Springer-Verlag, New York-Berlin, (1982).

[6] - Endomorphism rings and submodules of finite rank torsion-free abelian groups, Rocky Mountain J. Math., 12 (2) (1982), 241-256.

[7] D. M. Arnold and L. Lady, Endomorphism rings and direct sums of torsion-free abelian groups, Trans. Amer. Math. Soc., 211 (1975), 225-237.

[8] D. M. Arnold and C. E. Murley, Abelian groups $A$ such that $\operatorname{Hom}(A, \cdot)$ preserves direct sums of copies of $A$, Pacific J. Math., 56 (1) (1975), 7-20.

[9] G. Azumaya, Some Aspects of Fuller's Theorem, Lecture Notes in Mathematics, vol. 700, Springer-Verlag, New York-Berlin, (1979), 34-45. 
[10] N. Dugas and T. G. Faticoni, Cotorsion-free abelian groups cotorsion as modules over their endomorphism rings, to appear in the Proceedings of the Abelian Group Conference at Curacao, August 18-14, (1991).

[11] T. G. Faticoni, Examples of torsion-free abelian groups torsion as modules over their endomorphism rings, to appear in the Comm. Algebra.

[12] _ Gabriel filters on the endomorphism ring of a torsion-free abelian group, Comm. Algebra, 18 (9) (1990), 2841-2883.

[13] _ On the lattice of right ideals of the endomorphism rings of an abelian group, Bull. Austral. Math. Soc., 38 (2) (1988), 273-291.

[14] T. G. Faticoni and H. Pat Goeters, Examples of torsion-free groups flat as modules over their endomorphism rings, Comm. Algebra, 19 (1) (1991), 1-28.

[15] L. Fuchs, Infinite Abelian Groups I, II, Academic Press, New York-London, (1969, 1970).

[16] K. R. Fuller, Density and equivalence, J. Algebra, 29 (1974), 528-550.

[17] J. L. Garcia and M. Saorin, Endomorphism rings and category equivalences, J. Algebra, 127 (1) (1989), 182-205.

[18] J. Hausen, Modules with the summand intersection property, Comm. Algebra, 17 (1989), 135-148.

[19] M. Sato, Fuller's Theorem on equivalence, J. Algebra, 52 (1978), 274-284.

[20] B. Stenstrom, Rings of Quotients, Die Grundlehren der mathematischen Wissenschaften, vol. 217, Springer-Verlag, New York-Berlin, (1975).

[21] R. Wisbauer, Local-global results for modules over algebras and Azumaya rings, J. Algebra, 135 (2) (1990), 440-455.

Received April 30, 1991 and in revised form October 29, 1991.

FORDHAM UNIVERSITY

BRONX, NY 10458 


\title{
PACIFIC JOURNAL OF MATHEMATICS
}

\author{
Founded by
}

E. F. BECKENBACH (1906-1982) F. WoLF (1904-1989)

\section{EDITORS}

$\begin{array}{ll}\begin{array}{l}\text { V. S. VARADARAJAN } \\ \text { (Managing Editor) }\end{array} & \begin{array}{l}\text { NiCHOLAS ERCOLANI } \\ \text { University of Arizona }\end{array} \\ \text { University of California } & \text { Tucson, AZ 85721 } \\ \text { Los Angeles, CA 90024-1555 } & \text { ercolani@math.arizona.edu } \\ \text { vsv@math.ucla.edu } & \text { R. FinN } \\ \text { HERBERT CLEMENS } & \text { Stanford University } \\ \text { University of Utah } & \text { Stanford, CA 94305 } \\ \text { Salt Lake City, UT 84112 } & \text { finn@gauss.stanford.edu } \\ \text { clemens@math.utah.edu } & \text { VAUGHAN F. R. JoNEs } \\ \text { F. MichAEL CHRIsT } & \text { University of California } \\ \text { University of California } & \text { Berkeley, CA 94720 } \\ \text { Los Angeles, CA 90024-1555 } & \text { vfr@math.berkeley.edu } \\ \text { christ@math.ucla.edu } & \text { STEVEN KERCKHoFF } \\ \text { THomas ENRIGHT } & \text { Stanford University } \\ \text { University of California, San Diego } & \text { Stanford, CA 94305 } \\ \text { La Jolla, CA 92093 } & \text { spk@gauss.stanford.edu } \\ \text { tenright@ucsd.edu } & \end{array}$

\author{
C. C. MOORE \\ University of California \\ Berkeley, CA 94720
}

MaRTin ScharlemanN

University of California

Santa Barbara, CA 93106

mgscharl@henri.ucsb.edu

\author{
HAROLD STARK \\ University of California, San Diego \\ La Jolla, CA 92093
}

\begin{tabular}{ll}
\multicolumn{1}{c}{ SUPPORTING } & INSTITUTIONS \\
UNIVERSITY OF ARIZONA & UNIVERSITY OF OREGON \\
UNIVERSITY OF BRITISH COLUMBIA & UNIVERSITY OF SOUTHERN CALIFORNIA \\
CALIFORNIA INSTITUTE OF TECHNOLOGY & STANFORD UNIVERSITY \\
UNIVERSITY OF CALIFORNIA & UNIVERSITY OF HAWAII \\
MONTANA STATE UNIVERSITY & UNIVERSITY OF TOKYO \\
UNIVERSITY OF NEVADA, RENO & UNIVERSITY OF UTAH \\
NEW MEXICO STATE UNIVERSITY & WASHINGTON STATE UNIVERSITY \\
OREGON STATE UNIVERSITY & UNIVERSITY OF WASHINGTON
\end{tabular}

The Supporting Institutions listed above contribute to the cost of publication of this Journal, but they are not owners or publishers and have no responsibility for its content or policies.

Mathematical papers intended for publication in the Pacific Journal of Mathematics should be in typed form or offset-reproduced (not dittoed), double spaced with large margins. Please do not use built up fractions in the text of the manuscript. However, you may use them in the displayed equations. Underline Greek letters in red, German in green, and script in blue. The first paragraph must be capable of being used separately as a synopsis of the entire paper. In particular it should contain no bibliographic references. Please propose a heading for the odd numbered pages of less than 35 characters. Manuscripts, in triplicate, may be sent to any one of the editors. Please classify according to the 1991 Mathematics Subject Classification scheme which can be found in the December index volumes of Mathematical Reviews. Supply name and address of author to whom proofs should be sent. All other communications should be addressed to the managing editor, or Julie Speckart, University of California, Los Angeles, California 90024-1555.

There are page-charges associated with articles appearing in the Pacific Journal of Mathematics. These charges are expected to be paid by the author's University, Government Agency or Company. If the author or authors do not have access to such Institutional support these charges are waived. Single authors will receive 50 free reprints; joint authors will receive a total of 100 free reprints. Additional copies may be obtained at cost in multiples of 50 .

The Pacific Journal of Mathematics (ISSN 0030-8730) is published monthly except for July and August. Regular subscription rate: $\$ 190.00$ a year (10 issues). Special rate: $\$ 95.00$ a year to individual members of supporting institutions.

Subscriptions, orders for numbers issued in the last three calendar years, and changes of address should be sent to Pacific Journal of Mathematics, P.O. Box 969, Carmel Valley, CA 93924, U.S.A. Old back numbers obtainable from Kraus Periodicals Co., Route 100, Millwood, NY 10546.

The Pacific Journal of Mathematics at P.O. Box 969, Carmel Valley, CA 93924 (ISSN 0030-8730) is published monthly except for July and August. Second-class postage paid at Carmel Valley, California 93924, and additional mailing offices. Postmaster: send address changes to Pacific Journal of Mathematics, P.O. Box 969, Carmel Valley, CA 93924.

PUBLISHED BY PACIFIC JOURNAL OF MATHEMATICS, A NON-PROFIT CORPORATION Copyright $(\mathcal{1} 1993$ by Pacific Journal of Mathematics 


\section{PACIFIC JOURNAL OF MATHEMATICS}

Volume $157 \quad$ No. $2 \quad$ February 1993

Strong integral summability and the Stone-Čech compactification of the half-line

JEFF CONNOR and MARY ANNE SWARDSON

The endlich Baer splitting property

225

THEODORE GERARD FATICONI

The formal group of the Jacobian of an algebraic curve

MARGARET N. FREIJE

Concordances of metrics of positive scalar curvature

PAWEL GAJER

Explicit construction of certain split extensions of number fields and constructing cyclic classfields

STANLEY JOSEPH GURAK

Asymptotically free families of random unitaries in symmetric groups

ALEXANDRU MiHAi NiCA

On purifiable subgroups and the intersection problem

TAKASHI OKUYAMA

On the incidence cycles of a curve: some geometric interpretations

LUCIANA RAMELLA

On some explicit formulas in the theory of Weil representation

R. RANGA RAO

An analytic family of uniformly bounded representations of a free product of 373 discrete groups

JANUSZ WYSOCZAŃSKI

Errata: "Dentability, trees, and Dunford-Pettis operators on $L_{1}$ "

MARIA GIRARDI and ZHIBAO HU

Errata: "Poincaré cobordism exact sequences and characterisation" 